\title{
MedienPädagogik
}

Zeitschrift für Theorie und Praxis der Medienbildung

www.medienpaed.com

\section{Digitales mehrsprachiges Lernen bei neu zugewanderten Schülerinnen und Schüler der Grundschule}

\author{
Ergebnisse des dritten Design-Zyklus zum Einsatz audio-digitaler Stifte in \\ mehrsprachigen Rezeptionsprozessen
}

Juliane Dube

\begin{abstract}
Zusammenfassung
Wenngleich in den vergangenen 30 Jahren zum Für und Wider von digitalen Medien in der Schule zahlreiche Studien erschienen sind, generiert die ständige Weiter- und Neuentwicklung dieser in einem sich ebenfalls stetig verändernden Bildungsgefüge immer wieder neue Fragen. Für die Grundschule gilt es u.a. aktuell z.B. den Einsatz digitaler Formen wie (enhanced) eBooks oder Bilderbuch-Apps sowie digitale Audiostifte zur barrierefreien Rezeption von (Bilder-)Büchern aus deutschdidaktischer Perspektive stärker in den Blick zu nehmen. Ziel des Projektes MesH (Digitale Medien und sprachliche Heterogenität) war es audio-digitale Lernumgebungen zu mehrsprachigen Bilderbüchern zu entwickeln und diese sowohl im Labor-als auch im Schulsetting zu evaluieren (April 2017 - Juni 2018). Im vorliegenden Beitrag sollen die Ergebnisse des dritten Design-Zyklus vorgestellt werden, der sich mit der Frage beschäftigte, ob und inwieweit der Einsatz audio-digitaler Stifte als eine Form des computer assisted language learning (CALL) die Lernenden nicht nur zum Lesen motiviert, sondern auch eine Binnendifferenzierung im offenen Unterricht erlaubt, die den individuellen Stärken und Schwächen der mehrsprachigen Lernenden Rechnung trägt.
\end{abstract}

Digital multilingual learning with new immigrated students of the elementary school. Results of the third design cycle for the use of audio-digital pens in multilingual reception processes

\begin{abstract}
In the past 30 years a lot of articles about the pro and cons of digital media in school were publicated. New questions are generating by the constant development of changing educational system and the ongoing of digital media. Currently the use of (enhanced) eBooks, picture book apps and digital audio pens in primary schools will be discussed in german didactic. The aim of the project Digital Media and Linguistic Heterogeneity was to construct and to evaluate an audio digital learning environment with multilingual picture
\end{abstract}


books (April 2017 - June 2018). In this article the results of the third design cycle will be shown. On one hand the question of these circle was if and how far computer assisted language learning (CALL) with digital audio pens motivate pupils to read and on the other hand, if it creates options for individual learning.

\section{Einleitung}

Mit der wachsenden Etablierung einer Deutsch als Zweitsprachendidaktik gibt es inzwischen zahlreiche methodische Anregungen für die Gestaltung von Lernumgebungen in mehrsprachigen Klassen (u.a. Belke 2003; Engin 2004; Hoffmann und Weis 2011; Kalkavan-Aydin 2015). Ideen zur Gestaltung komplexerer Lernsituationen z.B. durch die Arbeit mit inter- und intralingualen Gedichten, Kurzgeschichten und Bilderbüchern zur Förderung von normgerechter Grammatik sowie der Anbahnung von Lese- und Textkompetenz, wie sie inzwischen zunehmend auch in der fachdidaktischen Literatur formuliert werden (Briddigkeit et al. 2004; Dohrn 2006; Belke 2009; Gawlitzek und Kümmerling-Meibauer 2013; Ballis, Pescher und Schuler 2019), finden sich jedoch nur selten in der Praxis. Als Ursachen für den kaum genutzten Einsatz literarischer Texte führen Lernpersonen neben der Komplexität der Texte vor allem die hohe sprachliche Diversität in den Lerngruppen an. Für eine längere Textarbeit werden zudem die hohe Fluktuation und die unzureichende Zeit für die Förderung angegeben (Dube und Gürsoy 2018).

Dabei bieten z.B. mehrsprachige Bilderbücher Anknüpfungspunkte an eine Vielzahl von schriftlichen, mündlichen und überfachlichen Lernbereichen (ausführlich dazu ebd.):

\section{Fördermöglichkeiten im Bereich Mündlichkeit:}

- phonologische Bewusstheit; Prosodie und Sprachrhythmus

- Sprachaufmerksamkeit und Sprachbewusstheit (Language Awareness)

- kommunikative Fähigkeiten (in der interaktiven Vorlesesituation)

\section{Fördermöglichkeiten im Bereich Schriftlichkeit:}

- Reflexion von Schriftlichkeit

- Nachdenken und Sprechen über eigene Spracherfahrungen (Metakommunikation)

- Wortschatzarbeit

- narrative Satz- und Textmuster

- textsortenspezifische Sprach- und Textstrukturen

- Funktion und Wirkung sprachlicher Stilmittel

- Lesemotivation 
- Lese- und Bildverstehen

- literarisches Lernen (Perspektivübernahme, etc.)

\section{Fördermöglichkeiten im überfachlichen Bereich:}

- Interkulturelle Kompetenz

Mehrsprachige Bilderbücher ermöglichen folglich nicht nur Situationen für innerfachliches Lernen z.B. im Bereich der Grammatik, sondern stellen auch Anschlussmöglichkeiten für ausserfachliches Handeln in Bezug auf die thematische Ausrichtung des Buches bereit. Hierzu schliessen die in Bilderbüchern verhandelten Themen an die Alltags- und Lebenswelt sowie die Erfahrungen und Interessen der Lernenden an. Die Erkenntnisse zum Einsatz des Bilderbuches und zur Gestaltung von Vorlesesituationen in Regelklassen (u.a. Spinner 2004; Möbius 2008; Kruse 2012) gilt es jedoch vor dem Hintergrund der hohen Diversität in Internationalen Förderklassen ${ }^{1}$ verstärkt zu reflektieren. Eine besondere Berücksichtigung sollten die Herkunftssprachen, die Sprachkompetenzen in der Zielsprache und die Schulerfahrungen der Lernenden erfahren, wenn es um zentrale Lerntätigkeiten des Erfahrens, Erklärens, Wiederholens und Vertiefens beim motivierenden Umgang mit dem Bilderbuch für alle Lernenden geht. Inwieweit der Einsatz audio-digitaler Stifte als eine Form des computer assisted language learning (CALL) die Lernenden nicht nur motiviert, sondern auch eine Binnendifferenzierung des offenen Unterrichts erlaubt, die den individuellen Stärken und Schwächen der Lernenden Rechnung trägt, gilt es noch zu prüfen.

\section{Audio-digitale Stifte als Unterstützungsmedium in der Leseförderung}

Tipto $^{\circledast}$ und tiptoi create ${ }^{\circledast}$ (Ravensburger), Bookii ${ }^{\circledast}$ und TING $^{\circledast}$ (Tessloff-Verlag) sowie Anybook Reader ${ }^{\circledast}$ (Franklin Electronic Publishers $\mathrm{GmbH}$ ) sind die Namen von Audiostiften, die es ihren Nutzerinnen und Nutzern ermöglichen, Informationen aus Büchern nicht nur zu lesen, sondern diese auch zu hören. Dazu tippen diese mit dem Stift auf markierte Stellen im Buch. Mithilfe des optischen Sensors der Stifte werden dadurch - analog wie bei einem Strich- oder QR-Code - hinterlegte oder selbstständig erstellte Hörtexte und Geräusche zu Texten und Abbildungen abgespielt oder über eine Bluetooth-Verbindung mit Smartphone, Tablet und PC Videos und weiterführende Links zugänglich.

1 Der Begriff Internationale Förderklassen wird in NRW synonym zu den Bezeichnungen Seiteneinstiegsklasse und Internationale Vorbereitungsklasse (IVK) verwendet. Dieser reine Sprachunterricht umfasst ca. zehn bis zwölf Unterrichtsstunden und die Verweildauer der Schülerinnen und Schüler darf i.d.R. einen Zeitraum von zwei Jahren nicht überschreiten. Die konkrete Organisation der Klassen hängt vom jeweiligen Standort und den individuellen Möglichkeiten der Schule ab. 


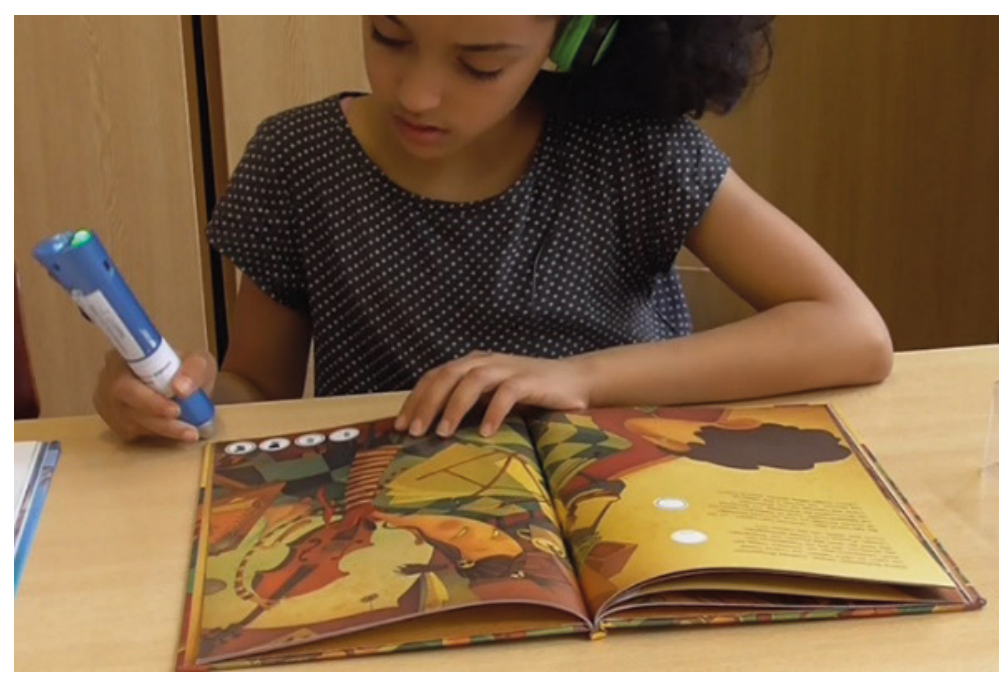

Abb. 1.: Schülerin mit präpariertem Bilderbuch und AnyBook-Reader ${ }^{\circledR}$.

Wie die Verkaufszahlen der letzten Jahre belegen, nach denen «sprechende» Stifte in etwa in jedem zweiten Haushalt mit Kindern zwischen vier und acht Jahren zu finden sind, ist die Ergänzung schriftlicher Medien um vielseitige Audiodateien ein Erfolgsmodell. ${ }^{2}$

Ursprünglich für den motivierenden Zugang zu Printmedien konzipiert, gibt es vom Marktführer tiptoi ${ }^{\circledR}$ (Ravensburger) heute auch Brettspiele, Globen und Spielfiguren, die mit dem Audiostift kombiniert werden können. Während tiptoi ${ }^{\circledR}$-Stifte mit ihren Begleitmaterialien vor allem in heimischen Wohnzimmern zum Einsatz kommen, gestalten andere Anbieter Audiostifte und Begleitmaterialien zunehmend auch für den Bildungsbereich. So wird inzwischen an vielen Schulen in NRW mit Ting ${ }^{\circledR}$ Stiften (Tessloff Verlag) und dazugehörigen vorgefertigten Bildungsmaterialien zum Zielsprachenerwerb gearbeitet. Ein differenzierteren Einsatz ermöglichen hingegen die Audiostifte von Anybook ${ }^{\circledast}$ (Franklin Electronic Publishers $\mathrm{GmbH}$ ) und Bookii (Tessloff Verlag), da sie den Lehrpersonen und Sprachanfängerinnen und -anfängern auch die spontane auditive Gestaltung jeglicher Printmaterialien ermöglichen (vgl. Abb. 2).

2 «Ravensburger wächst dank Lernstift Tiptoi»<https://www.wiwo.de/unternehmen/handel/vor-der-spielemesse-in-essen-ravensburger-waechst-dank-lernstift-tiptoi/19326944.html>, am 24.10.2019. 


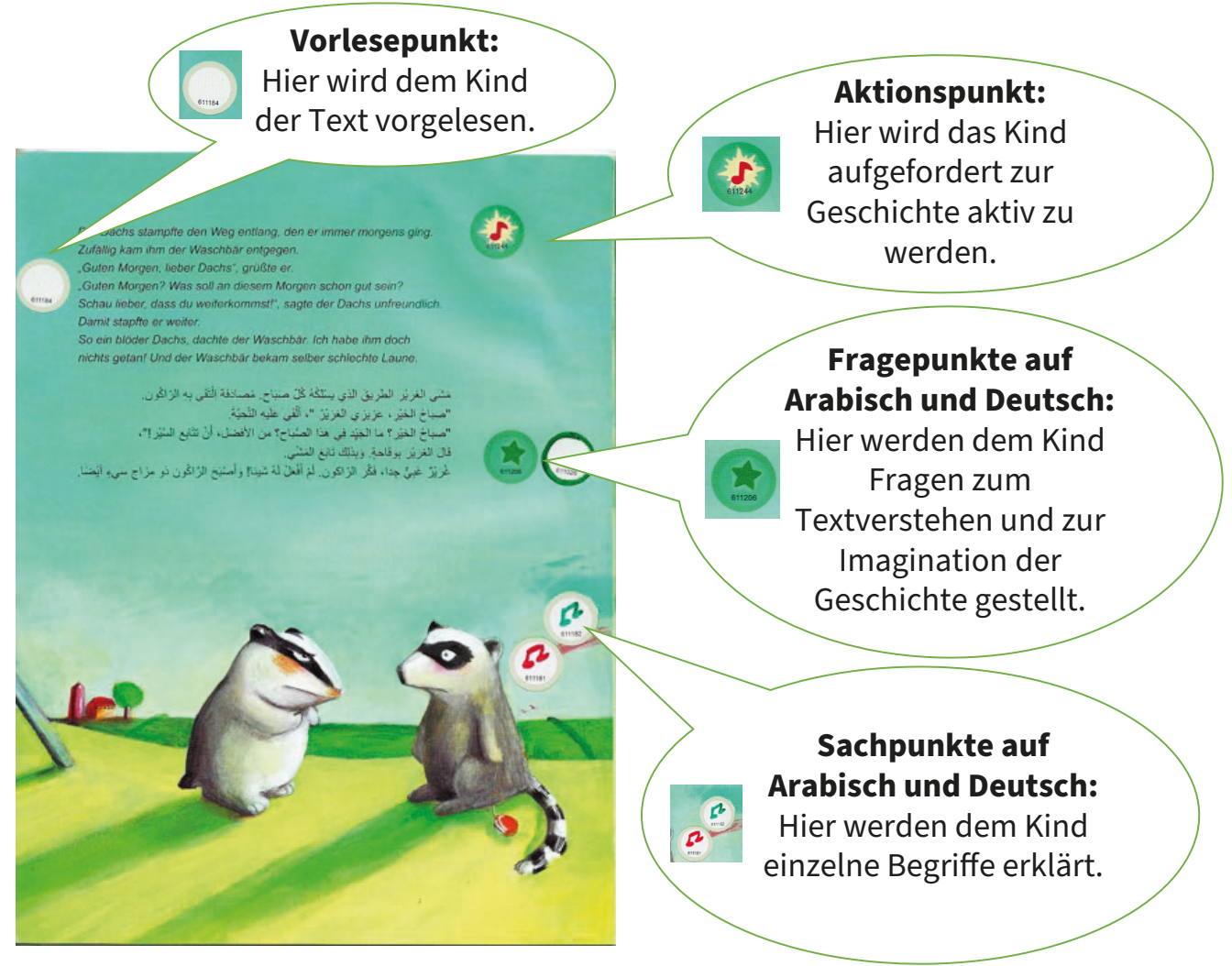

Abb. 2.: Eigengestaltung von Bilderbuchseiten mit den Audiostickern des Anybook-Readers.

Das individuelle Besprechen der zum Audiostift gehörenden Audiosticker ermöglicht folglich das Erstellen vielfältiger Audiodateien. Mit Bezug zur mehrsprachigen Lerngruppe in Internationalen Förderklassen bieten sich folgende (mehrsprachige) Inhalte an:

- Übersetzungen von einzelnen Sätzen und Textabschnitten,

- ergänzende Hinweise zu Bildern und Texten,

z.B. Das ist der Waschbär. Der Waschbär ist ein guter Kletterer. Der Waschbär lebt meist auf Bäumen.

- Begriffsklärungen,

- Handlungs- und Produktionsimpulse, z.B. Kannst du auch ein Lied pfeifen? Versuche es.

- Fragen zur Vorwissensaktivierung, z.B. Wie feierst du Geburtstag?

- Fragen zum Text, z.B. Welche Tiere hat der Dachs bisher getroffen?

- Antworten zum Text, z.B. Der Dachs hat bisher den Waschbären und den Elch getroffen. 
- Spiele und Rätsel, z.B. Ordne die Wortkarten den Bildern zu. Kontrolliere mit dem Stift.

- Fragen zur Imagination, z.B. Wovon könnte die Geschichte handeln?

- Anregungen zur Sprachreflexion, z.B. zum Wortschatz (Dachs - faucht; Eichhörnchen - pfeift, Waschbär - knurrt)

- Geräusche zur akustischen Untermalung der Bilder,

- freie Audiosticker zur Gestaltung eigener Sprachaufnahmen durch die Lernenden.

Eine dazugehörige Software ermöglicht es zudem, Beiträge auf weitere Stifte zu duplizieren bzw. Inhalte aus dem Internet aufzuspielen.

\section{Forschungsdesign im Projekt MesH}

Im interdisziplinären Forschungsprojekt Digitale Medien und sprachliche Heterogenität $(\mathrm{MesH})^{3}$ entwickelten das Institut für Germanistik, das Institut für Deutsch als Zweit- und Fremdsprache und das Institut für Psychologie der Universität Duisburg Essen audio-digitale Lernumgebungen zu mehrsprachigen Bilderbüchern und begleiteten deren Umsetzung empirisch. Entsprechend der aus den Bedürfnissen der Praxis heraus entstandenen Fragestellung, wie komplexere literarische Texte auch in Klassen mit neu Zugewanderten eingesetzt werden können, wurde als methodischkonzeptioneller Rahmen das Forschungsformat der Fachdidaktischen Entwicklungsforschung gewählt (Dube 2019a, Dube und Hußmann 2019). In diesem «Format fachdidaktischer Forschung» (GFD-Positionspapier 2016) stehen die Entwicklung von Lernarrangements, die Beforschung von Lernprozessen und die Weiterentwicklung von lokalen Theorien im Kontext eines zyklisch angelegten Forschungsprozesses gleichberechtigt nebeneinander. Fachdidaktische Entwicklungsforschung soll dabei einen exemplarischen Beitrag zur Lösung eines didaktischen Problems leisten und dadurch Theorien hervorbringen. Diese sind zwar stets kontextgebunden und damit lokal, aber so ausgearbeitet, dass sie für eine übergreifende Praxis relevant sind. Fragen wie «Was funktioniert, warum, wann und für wen` stehen im Zentrum des Interesses (Cobb et al. 2003, 9). Für die Beantwortung der übergeordneten Forschungsfrage sollten im Zeitraum von April 2017 - Juni 2018 vier Design-Zyklen umgesetzt werden (vgl. Abb. 3).

3 Finanziert wurde das Projekt über das Programm zur Förderung des exzellenten wissenschaftlichen Nachwuchses der Universität Duisburg-Essen. Die Finanzierung der Audiostifte wurde von der Bertelsmann Stiftung übernommen. 


\begin{tabular}{|l|l|l|l|l|}
\hline \multicolumn{1}{|c|}{ 1. Zyklus } & \multicolumn{1}{c|}{ 2. Zyklus } \\
\hline Einzelförderung
\end{tabular}

Abb. 3.: Design-Zyklen im Projekt MesH.

In einem ersten Zyklus wurden die audio-digitalen Lernarrangements zusammen mit (mehrsprachigen) Studierenden im Rahmen von Theorie-Praxis-Seminaren an der Universität Duisburg-Essen erfahrungsorientiert entwickelt und in je drei Einzelsitzungen, in denen zwei Studierende die Funktionen von Leitung und Beobachtung übernahmen, erprobt. Die Fragen auf Forschungs- und Entwicklungsebene waren zunächst sehr allgemein formuliert. Sie bezogen sich einerseits auf Veränderungen im Bereich sprachlicher und literarischer Prozesse in der Zielsprache und andererseits auf die Chancen und Herausforderungen des kombinierten Einsatzes von digitalem Audiostift und mehrsprachigen Bilderbuch.

Für die Beobachtung der 35 Projektteilnehmenden, die auf drei Grundschulen verteilt waren, wurde ein hochstrukturiertes Beobachtungsprotokoll von der Projektleitung erstellt.

Die Ergebnisse aus den Beobachtungsprotokollen, dem diskursiven Erfahrungsaustausch sowie den schriftlichen Reflexionsberichten der Studierenden bildeten die Basis für die erste Überarbeitungsphase des Lernsettings. Hierbei standen insbesondere die inhaltliche Ausgestaltung der Audiosticker im Fokus mit dem Ziel, die sprachlichen und literarischen Lernprozesse im Rezeptionsprozess enger aufeinander abzustimmen und die Mehrsprachigkeit der Lernenden noch stärker einzubeziehen. Zusammengeführt wurden die Überlegungen zur Überarbeitung der Lernumgebung in dem Konzept der Sprachintegrativen Leseförderung (Dube 2019b), welches fortan als Basis für die (Weiter-)entwicklung prototypischer Lernumgebungen in den folgenden Zyklen diente.

Im zweiten Zyklus wurden die überarbeiteten Lernumgebungen erneut von zwei Studierenden in Einzelsitzungen eingesetzt und die daraus resultierenden Beobachtungen dokumentiert. Eine Konkretisierung der Fragestellung erfolgte in Bezug auf die Eingrenzung auf Lesekompetenz und morphosyntaktische Sprachfähigkeiten im Rahmen des Konzepts der Sprachintegrativen Leseförderung. Abermals übernahmen die Studierenden die Rolle von Leitung und Beobachtung. Auf Grundlage der überarbeiteten Beobachtungsprotokolle, dem diskursiven Austausch und den schriftlichen 
Reflexionsberichten der Studierenden konnten am Ende des zweiten Zyklus erste vorläufige Design-Prinzipien formuliert werden. Hierzu zählen: 1. Bilderbuchauswahl unter ganzheitlicher Perspektive, 2. Einbindung der Herkunftssprachen und 3. Entwicklungsproximale Gestaltung authentischer Begleitaufgaben (Dube 2019b, 162f). Aufgrund der nach wie vor beobachteten fehlenden Engführung des Leseprozesses lag der Schwerpunkt des dritten Zyklus in der Ausschärfung der Design-Prinzipien mit Schwerpunkt auf den Bereich der Lesemotivation. Hierzu wurden im Konzept der Sprachintegrativen Leseförderung Ausführungen zum Vorlesegespräch (Spinner 2004), zum Sehgespräch (Möbius 2008), zum Hörgespräch (Hüttis-Graff 2008) und zur intermedialen Lektüre (Kruse 2012) stärker berücksichtigt. Da die Lernumgebungen bisher nur in Einzelsitzungen eingesetzt worden, in denen die Schülerinnen und Schüler mal mehr und mal weniger eng begleitet wurden, sollten die Lernumgebungen nun auch so adaptiert werden, dass die Lernenden zukünftig möglichst selbstständig mit diesen arbeiten können.

\section{Fragestellung und Methodik}

Zentrale Fragestellungen der systematischen Unterrichtsbeobachtung im dritten Design-Zyklus waren folglich:

1. Frage auf Forschungsebene:

Welchen Einfluss hat die Erweiterung des Konzepts der Sprachintegrativen Leseförderung um Reflexions- und Gesprächsimpulse auf den audiostiftgestützten Rezeptionsprozess und die Lesemotivation in der Zielsprache bei neu zugewanderten Schülerinnen und Schüler?

2. Frage auf Entwicklungsebene:

Wie muss die Lernumgebung mit mehrsprachigen Bilderbüchern und audio-digitalen Stiften zur Sprachintegrativen Leseförderung konzipiert sein, damit neu zugewanderte Schülerinnen und Schüler selbstständig mit diesen arbeiten können?

Mit dem Ziel auf Forschungsebene den audiostiftgestützten Rezeptionsprozess sowie die Lesemotivation und auf Entwicklungsebene verhaltensnahe Beschreibungen bzw. Ereignisse der mediengestützten Mikroprozesse auf Ebene der «Sichtstruktur» beschreiben zu können, wurde ein exploratives Untersuchungsdesign gewählt. Im Zentrum dessen stand ein strukturierter Beobachtungsbogen mit fünf hoch 
inferenten Kategorien und 31 Items. ${ }^{4}$ Für die Beobachtung der Kategorien: Technische Handhabung des Audiostiftes (4 Items), Umgang mit Stift und Bilderbuch (10 Items), Leseverhalten (3 Items), Arbeit mit den begleitenden Leseaufgaben (6 Items) und Lesemotivation (8 Items) standen Pole von vier (trifft voll und ganz zu) bis eins (trifft nicht zu) für eine geschulte studentische Mitarbeiterin ${ }^{5}$ zur Auswahl. In einem Freifeld sollte diese zudem über die vorgegebenen Items hinausgehende Beobachtungen wie u.a. Schülerfragen, Lernatmosphäre, Unterrichtsstörungen etc. dokumentieren.

Zusätzlich wurde die Förderstunde mit einer Kamera aufgezeichnet, um den Interpretationsprozess zu den Beobachtungsprotokollen zu stützen. Für den leichteren Abgleich der Situationen wurden in Anlehnung an ein Eventsampling auf dem Beobachtungsprotokoll ebenfalls die Zeiten der beobachteten Handlungen notiert. Im Mittelpunkt der Beobachtung standen pro Sitzung zwei Probandinnen und Probanden, deren Verhalten in der Lernumgebung qualitativ (Ausprägung) und quantitativ (Anzahl) erfasst wurde.

\begin{tabular}{|l}
\hline Skala Lesemotivation \\
(++ trifft voll und ganz zu, + trifft eher zu, - trifft weniger zu, -- trifft nicht zu, 99 - k.A.) \\
\hline Item \\
\hline gegenstandsbezogenen Lesemotivation (mehrsprachige Bilderbücher) \\
Der Schüler/die Schülerin reagiert positiv auf das Lesen der mehrsprachigen Bilderbücher. (An- \\
kerbeispiel: Der TN/die TN äussert sich positiv verbal «Tolles Buch», gestisch (Daumen hoch, \\
blättert vor Stundenbeginn im Bilderbuch) oder mimisch (Lachen) \\
\hline erlebnisbezogene Lesemotivation (Text) \\
Der Schüler/die Schülerin reagiert positiv auf das Gelesene. (Ankerbeispiel: Der TN/die TN äussert \\
Freude über das Gelesene: «Das ist witzig», «Das les`ich noch einmal») \\
\hline erlebnisbezogene Lesemotivation (Audiosticker) \\
Der Schüler/die Schülerin reagiert positiv auf die Inhalte der Audiosticker. (Ankerbeispiel: Der \\
TN/die TN äussert verbal «Das ist witzig», oder mimisch (Lachen) Freude über die Inhalte der \\
Audiosticker)
\end{tabular}

4 Für die passive Beobachtung mit den hochstrukturierten Beobachtungsbögen wurden die deduktiv und induktiv gewählten Merkmale fachunspezifisch operationalisiert, d.h. weder fachspezifische Besonderheiten noch spezifische fachwissenschaftliche oder fachdidaktische Kenntnisse waren auf Seiten der Raterinnen nötig. Das Kategoriensystem wurde nach dem ersten Zyklus überarbeitet und einem Reliabilitätstest unterzogen. Dazu wurden von der Projektleitung und einer studentischen Hilfskraft je fünf Fördersitzungen kodiert und die Ergebnisse miteinander verglichen. Die so ermittelte Intercoder-Reliabiliät betrug 0,85 (nach Holsti), wonach der Beobachtungsbogen als präzise und verlässlich genug angesehen werden kann. Die Reliabilität der überarbeiteten und ergänzten Beobachtungsbogen am Ende des zweiten Zyklus wurde ebenfalls anhand von fünf Fördersitzungen des zweiten Zyklus geprüft. Die Intercoder-Reliabiliät betrug 0,86 (nach Holsti).

5 Um Wahrnehmungs- und Deutungsfehler zu vermeiden, wurden beide Raterinnen anhand älterer Videos aus Einzelsitzungen zu Zyklus zwei in der Anwendung des Protokolls im Rahmen einer zweistündigen Einführung trainiert. 
leistungsbezogene Lesemotivation

Der Schüler/die Schülerin zeigt Interesse, die eigene Lesekompetenz zu verbessern. (Ankerbeispiel: Der TN/die TN äussert sich verbal zum Fortschritt des eigenen Leseprozesses oder dem Bearbeitungsstand der Leseaufgaben)

kommunikationsbezogene Lesemotivation (Text)

Der Schüler/die Schülerin zeigt das Bedürfnis sich über das Gelesene mit der Gruppe auszutauschen. (Ankerbeispiel: Der TN/die TN unterbricht den Leseprozess, um der LP oder den anderen TN vom Gelesenen zu berichten).

\section{kommunikationsbezogene Lesemotivation (Audiosticker)}

Der Schüler/die Schülerin zeigt das Bedürfnis sich über die Inhalte der Audiosticker mit der Gruppe auszutauschen. (Ankerbeispiel: Der TN/die TN unterbricht den Leseprozess, um der LP oder den anderen TN vom Inhalt der Audiosticker zu berichten).

$\ldots$

Tab. 1.: Auszug aus dem strukturierten Beobachtungsprotokoll.

Um die aus der nicht teilnehmenden Beobachtung gewonnenen Daten zu ergänzen bzw. strittige Stellen zur Diskussion zu stellen, wurden die Teilnehmenden nach der letzten Sitzung zudem einzeln interviewt. Für die ca. 20-minütigen leitfadengestützten Interviews wurden die Lernenden gebeten, ein bis zwei Bilderbücher auszuwählen. Entlang der Bilderbücher und der dort ergänzten Audiosticker wurde die Interviewsituation aufgebaut, die Fragen zum allgemeinen Projektablauf, zur konkreten Arbeit mit der mehrsprachigen audio-digitalen Lernumgebung, der Buchauswahl sowie zur Lesemotivation umfasste. Die Antworten der Befragten wurden inhaltsanalytisch nach Meuser und Nagel 2009 ausgewertet. ${ }^{6}$

Forschungsdesign und Gestaltung der mehrsprachigen digitalen Lernumgebung im dritten Design-Zyklus

Während die ersten Design-Zyklen im Projekt auf die Erprobung der digitalen Lernumgebung und der Herausarbeitung erster Design-Prinzipien für eine Sprachintegrative Leseförderung ausgerichtet waren, konzentrierten sich die Zyklen drei und vier auf die Ausschärfung dieser unter regulären schulischen Rahmenbedingungen (vgl. Abb. 3). Zyklus drei fokussierte dabei die stärkere Einbindung literaler Verstehensprozesse in das Lernsetting sowie die Selbstständigkeit des Rezeptionsprozesses. Der hohe Grad an Selbstständigkeit begründet sich auf der einen Seite aus lernpsychologischer Sicht, wonach Lernen als ein aktiv-konstruktiver, selbstgesteuerter

6 Wenngleich die Schülerinnen und Schüler im Projekt nicht in erster Linie als Experten mit speziellem Fachwissen anzusehen sind, welches nicht jedem in dem Handlungsfeld zugänglich wäre (Meuser und Nagel 2009, 467), sind sie dennoch Kenner der eingesetzten Lernumgebung, sodass sie im weitesten Sinne als »Experten« angesehen werden können.

Schülerin 004 ist auch zur Zeit der Interviews nicht mehr an der Schule zugegen. Schüler 002 kann aufgrund von Krankheit ebenfalls nicht befragt werden. 
Prozess verstanden wird, aus motivationspsychologischer Sicht, wonach schon geringe Wahlfreiheiten und die Möglichkeiten zur Selbstkontrolle die Motivation steigern (Munser-Kiefer 2014) und auf der anderen Seite aus schulpraktischer Sicht, wonach insbesondere im Unterricht mit Seiteneinsteigerinnen und -einsteigern verstärkt Phasen des individuellen Lernens umgesetzt werden sollten, um der vorherrschenden (sprachlichen) Heterogenität der Lernenden gerecht zu werden. Die durch das Lernsetting entstehende hohe Passung zwischen sprachlichen Fähigkeiten in der Ziel- und Herkunftssprache sowie dem Gegenstand des Bilderbuchs wurde ein hoher Anteil echter Lernzeit erwartet. Orientierung in den individuellen Arbeitsphasen boten nicht nur ein Lesepass, in dem die Lernenden Datum und Buchauswahl notierten, sondern auch ein wiederkehrender mehrsprachiger Audiotext, der in den Umgang mit Bilderbuch und Audiostift einführte.

Während Beobachtungen aus den ersten beiden Design-Zyklen zum freien Umgang mit dem Audiostift zeigten, dass das Hören des Textes im Vordergrund steht und die Sprach- und Leseaufgaben nur selten beachtet werden, wurden in der Einführung des dritten Design-Zyklus verstärkt Elemente aus der Vorlesepraxis aufgegriffen. Mit dem Ziel, die Selbstregulation des Rezeptionsprozesses zu unterstützen, wurden auch Hinweise zur Rezeptionssteuerung auf Audiostickern ergänzt. So sollte ein Umblättern erst nach dem Beantworten der Fragen bzw. Aufgaben auf den Audiostickern erfolgen. Zudem wurde auf das Mitlesen des Textes nicht nur in der Einführung zu Projektbeginn verwiesen, sondern auch immer wieder in den einzelnen Audioaufnahmen zu den Leseaufgaben im Bilderbuch.

\section{Beispiel für Hinweis zum Mitlesen auf einem Audiosticker:}

Welches Tier bekommt Ritter Winzig geschenkt? Lies auf der Seite noch einmal genau nach.

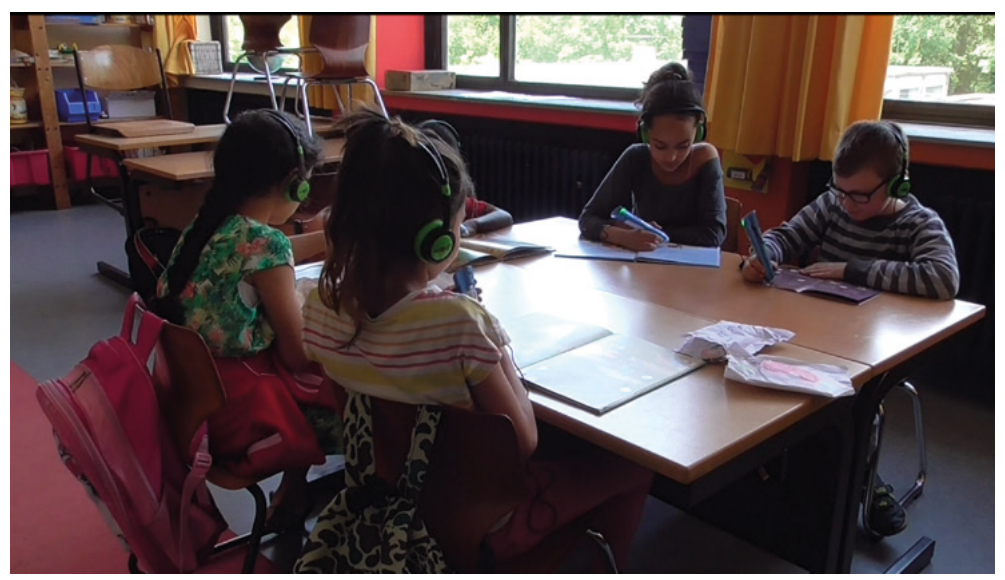

Abb. 4.: Schülerinnen und Schüler bei der selbstständigen Lektüre mit den Audiostiften (6:13, Kamera 2, 3. Sitzung). 
Die Schülerinnen und Schüler arbeiteten im dritten Design-Zyklus über 12 Wochen (März 2018 - Juni 2018) einmal wöchentlich in zwei Kleingruppen zusammen. ${ }^{7}$ Jede Kleingruppe bestand aus drei Schülerinnen und Schülern, einer Beobachterin und einer geschulten Förderlehrkraft ${ }^{8}$. Diese wöchentliche Förderstunde war dabei Teil des regulären schulischen DaZ-Förderunterrichts und wurde durch die Projektleitung oder eine studentische Hilfskraft angeleitet. Die Schülerinnen und Schüler, die nicht am Projekt teilnahmen, besuchten parallel zur Projektförderstunde den klassischen DaZ-Förderunterricht.

Im Zentrum der wöchentlichen Förderstunde stand ein mehrsprachiges Bilderbuch, welches durch Audiosticker des Anybook-Readers erweitert war. Die Auswahl der mehrsprachigen Bilderbücher umfasste u.a. folgende Werke:

- «Alis Nase» von Yekta Kopan (2013)

- «Der Regenbogenfisch» von Marcus Pfister (u.a. 2017)

- «Eine Kiste nichts» von Lena Hesse (2015)

- «Der Dachs hat heute schlechte Laune» von Moritz Petz (2015)

- «Ritter Winzig» von Andreas Völlinger (2016)

- «Wer hat mein Eis gegessen» von Rania Zaghir (2014)

Zu Beginn jeder Förderstunde wählten die Teilnehmenden ein präpariertes mehrsprachiges Bilderbuch zum Lesen aus. Die hierfür von der Projektleitung erstellte Auswahl basierte auf dem Lese- und Sprachstand der Lernenden und umfasste mindestens drei Bilderbücher pro Sprache. Nach den Eintragungen in den individuellen Lesepass begannen die Lernenden selbstständig mit dem audio-digitalen Leseprozess. Um die Lernenden zu einem intensiven Rezeptionsprozess zu motivieren, wurden die Bilderbücher um mehrsprachige Leseaufgaben ergänzt, die im Anschluss von den Teilnehmenden in der Zielsprache Deutsch beantwortet werden sollten. Die Aufgaben umfassten Fragen zum Leseverstehen und zur Lesegenauigkeit.

\section{Stichprobe}

Durchgeführt wurde die Studie an einer Grundschule, an der seit dem Schuljahr 2014/2015 rund 80 Kinder mit Zuwanderungsgeschichte teilintegrativ, d.h. einerseits in jahrgangsbezogen und andererseits in speziellen DaZ-Lerngruppen unterrichtet werden. Für das Projekt wurden sechs neu zugewanderte Kinder mit

7 Eine der Sitzungen musste aufgrund einer schulischen Veranstaltung ersatzlos entfallen.

8 Die Rolle der Förderlehrkraft übernahm die Projektleitung selbst oder eine geschulte studentische Hilfskraft. 
unterschiedlichen sprachlichen Kompetenzen in der Zielsprache Deutsch von den Lehrpersonen ausgewählt. ${ }^{9}$

\begin{tabular}{|l|l|l|l|l|l|}
\hline & & Alter & Herkunftssprache(n)/ Land & $\begin{array}{l}\text { Kontaktdauer } \\
\text { Zweitsprache } \\
\text { seit }\end{array}$ & $\begin{array}{l}\text { Globalurteil } \\
\text { Sprach- } \\
\text { niveau }\end{array}$ \\
\hline 001 & w & 9 & Arabisch, Türkisch (IRAN) & Aug. 2016 & A2 \\
\hline 002 & m & 8 & Polnisch (POLEN) & Aug. 2017 & A1 \\
\hline 003 & m & 9 & Englisch (GHANA) & Aug. 2016 & A2 \\
\hline 004 & w & 9 & Arabisch (SYRIEN) & Aug. 2017 & A1 \\
\hline 005 & w & 9 & Arabisch (IRAK) & Aug. 2016 & B1 \\
\hline 006 & w & 9 & Spanisch, Arabisch (SPANIEN) & Aug. 2016 & B1 \\
\hline
\end{tabular}

Tab. 2.: Übersicht Stichprobe dritter Design-Zyklus.

\section{Erste Ergebnisse der empirischen Begleitstudie auf Forschungsebene}

\section{Lesemotivation}

Ähnlich wie in anderen Studien auch, war bei den Kindern von Beginn an ein grosses Interesse an der Nutzung der eingeführten digitalen Medien zu beobachten. Bereits beim Auspacken der Audiostifte zeigten sie grosse Begeisterung und kommentierten den Prozess mit Zwischenrufen, wie «cool» (Schüler 003) oder «Jaaa, geil» (Schülerin 001). Zudem zeigten sie sich gegenseitig die Audiostifte. Dem Freitext des Beobachtungsprotokolls ist zu entnehmen, dass drei Schülerinnen während der Fördersitzungen bereits von Erfahrungen im Umgang mit ähnlichen Audiostiften im häuslichen Umfeld erzählten. Die Beschreibungen der Lernenden lassen darauf schliessen, dass in den Haushalten Audiostifte der Marke tiptoi ${ }^{\circledR}$ vorhanden sind.

Die Idee, den Audiostift für das Lesen von mehrsprachigen Bilderbüchern zu nutzen, wurde ebenfalls von allen Teilnehmenden als sehr positiv bewertet. Noch vor einer allgemeinen technischen Einführung wurden die ersten Audiosticker angespielt. Mit besonderem Zuspruch, wie in Form von «Wow, das Spanisch» (Schülerin 006) kommentierten die Lernenden die Mehrsprachigkeit der Bücher, die folglich neben der auditiven Lesehilfe als markanter Motivationsanker klassifiziert werden kann.

Die hohe Motivation für den audiogestützten Leseprozess trägt bei den sprachlich fortgeschrittenen Teilnehmenden bis zur letzten Sitzung. So zeigen die

9 Die Lehrpersonen wählten die Kinder für die Projektteilnahme nach den Vorgaben der Projektleitung unter der Berücksichtigung der Kriterien Sprachstand in der Zielsprache und Lesemotivation aus. Dabei differenzierten sie nach geringen und fortgeschrittenen Sprachkompetenzen in der Zielsprache Deutsch sowie wenig und hoher Lesemotivation. Die Einschätzung des Sprachniveaus basierte auf einem Globalurteil der betreuenden Lehrperson in der DaZ-Förderung. 
Beobachtungsprotokolle, dass diese Lernenden überwiegend positiv auf den wöchentlichen Projektbeginn sowie die Buchauswahl reagieren, keiner äusseren Ansprache bedürfen, um den Leseprozess zu beginnen bzw. fortzusetzen und diesen auch nur kurzzeitig unterbrechen. Nach dem ersten Lesen der zur Verfügung stehenden Bilderbücher entschliessen sie sich zudem, teilweise unaufgefordert, zur erneuten Lektüre.

Die hohe Lesemotivation der Lernenden zeigte sich auch durch den wiederkehrenden Wunsch, sich über das Gelesene auszutauschen. So belegten die Beobachtungen, dass alle Lernenden ab und an ihren Leseprozess unterbrachen, um auf witzige Textstellen und Audioaufnahmen hinzuweisen.

Die Aussagen der fortgeschrittenen Sprecherinnen und Sprecher bestätigen im leitfadengestützten Interview diese Beobachtungen. So betonen die Befragten, dass sie stets mit einem positiven Gefühl die Leseförderung besucht haben, und auch der kombinierte Einsatz von Stift und Bilderbuch Gefallen fand. Schülerin 005 verwies besonders auf die Möglichkeit, eigene Aufnahmen zu gestalten:

«Da war so coole Sachen, du kannst ein Heft da gab es, du sprichst auf die Heft, danach kannst du das hören, wie hast du es gesprochen» (Z: 17-18). ${ }^{10}$

Wenngleich bei schwächeren Sprecherinnen und Sprechern immer wieder auch Schwierigkeiten im Leseprozess beobachtet werden konnten, wird die Lesemotivation dieser kaum negativ beeinträchtigt. Allerdings verschiebt sich die Zieldimension hinsichtlich der überwiegenden Beschäftigung mit herkunftssprachlichen Texten, die überwiegend über die Audiofunktion des Stiftes rezipiert werden. Wie eng Lesemotivation und Leseverstehen korrelieren, zeigt sich auch in einer Interviewsequenz mit Schüler 003. Angesprochen auf die Buchauswahl erzählt Schüler 003 vom Missfallen des Buches «Eine Kiste nichts» von Lena Hesse, weil es da «nur» (Z: 10) um eine leere Kiste ging, die auch am Ende noch leer bleibt. Das literarische Potenzial dieser leeren Kiste, die im Verlauf des Bilderbuches unzählige Einsatzmöglichkeiten zeigt und damit viel mehr ist als «nur» eine Kiste wird von Schüler 003 offensichtlich nicht erfasst. Das Bilderbuch «Alis Nase» hingegen wird von ihm positiv besprochen, weil: «Alis Nase, das war gut, weil Ali hat ihre Nase gefunden» (Z: 12).

Wenngleich die Motivation im Projekt bei allen Lernenden überwiegend hoch war, konnten sich nur Schülerin 005 und Schülerin 001 den Einsatz des Audiostiftes im häuslichen Umfeld vorstellen.

10 Wenngleich nicht im Fokus der hier vorgestellten Ergebnisse sei dennoch auch auf die positive Bewertung der Mehrsprachigkeit in den Bilderbüchern verwiesen. Zwei Schülerinnen gaben an, noch nie ein Bilderbuch in ihrer Herkunftssprache gesehen zu haben, sodass sie nun noch mehr Lust hätten, dieses zu lesen. Ein anderes Mädchen fühlte sich zudem angeregt, eigene spanische Bilderbücher von zu Hause mitzubringen, um diese anderen zu zeigen. 


\section{Audiostiftgestützter Rezeptionsprozess des mehrsprachigen Bilderbuches}

Mit Ausnahme von Schülerin 004 begannen alle Kinder ihren Leseprozess mit den zielsprachlichen Texten. Anschliessend wechselten sie, mit Ausnahme von Schüler 002, der sich auch in den folgenden Sitzungen trotz grosser Verständnisschwierigkeiten wiederholt der Nutzung der Übersetzung verwehrte, in ihre Herkunftssprachen. Die Rezeptionsreihenfolge beider Sprachen wurde von den fortgeschrittenen Lernenden überwiegend beibehalten. Beim wiederholten Lesen des Bilderbuches griffen diese schliesslich nicht mehr auf die Übersetzungen zurück:

«Immer mach ich erst auf Deutsch und dann auf meine Muttersprache [Spanisch], aber jetzt hab ich immer nur auf Deutsch gelesen» (Schülerin 006, Z: 25-26).

Schwächere Lernende verweilten in ihrer Herkunftssprache (Schülerin 004), nutzten fast nur den deutschen Text (Schüler 002) oder wechselten nach einem ersten Durchgang in die Herkunftssprache, um dann anschliessend den zielsprachlichen Text zu lesen bzw. zu hören (Schüler 003).

Ungeachtet dieses ersten allgemeinen, explorativen Zugangs zum Bilderbuch unterschieden sich die beobachteten Textrezeptionen in Abhängigkeit vom Sprachniveau der Zielsprache jedoch bisweilen deutlich. So zeigten die fortgeschrittenen Lernerinnen 006, 005 und 001 mit jeder Sitzung einen zunehmend zielgerichteteren Umgang mit dem Audiostift bis sie am Projektende weder zum Lesen der deutschen noch der herkunftssprachlichen Texte auf den Stift als Unterstützungsmedium im Leseprozess angewiesen waren.

Dass eine stärkere Lenkung des Rezeptionsprozesses über geänderte und erweiterte Formulierungen bei den Audiostickern gelungen ist, belegen nicht nur die Beobachtungsnotizen, die einerseits nach Impulsen immer wieder ein Innehalten im Leseprozess, ein Zurückblättern und ein Suchen nach den gefragten Textstellen dokumentieren, sondern auch eine Reihe von Aussagen in den Einzelinterviews. So kann u.a. Schülerin 005 nach einem dreiwöchigen Abstand zur Lesesituation, den Handlungsverlauf zum Bilderbuch Eine Kiste nichts von Lena Hesse umfangreich wiedergeben und sich darüber hinaus auch an die weiterführenden Leseaufgabe erinnern:

«Eine Kiste nichts drin, glaub ich das heißt so. Da war ein Hühnchen und der Hase hat gebringt eine Kiste und sie fragt sich selber was kann da sein drin und da war nichts. Dann hat sie gefragt: ‘Warum ist die Kiste ohne drin?» Da hat der gesagt: «Weil das kannst du basteln so Sachen〉. Dann hat es geregnet und dann sind die da reingegangen. Das war Sommer ein bisschen warm, dann haben die so ein Haus gemacht und dann den Hühnchen hat eine Hose gebastelt und den Hase hat selber einen Hut gebastelt. [Bezug zur Leseaufgabe] Und da in 
ein Platz sagt sie: ‘Was kannst du noch was machen mit dem Karton?> Dann hab ich gedacht ein Hauskarton, das ist raus gegangen. Das war ein bisschen lustig.» (Z: 13-19).

\section{Erste Ergebnisse der empirischen Begleitstudie auf Entwicklungsebene}

\section{Praktikabilität - Umgang mit dem Hörstift}

Im Allgemeinen zeigten alle Schülerinnen und Schüler eine kompetente Mediennutzung. Sowohl das Abspielen und das eigenständige Erstellen von Aufnahmen bereitete keinem der Projektteilnehmenden Schwierigkeiten. ${ }^{11}$ Auch das Ein- und Ausschalten, das Anwählen der Audiosticker, die Regulierung der Lautstärke sowie das Verbinden mit den Kopfhörern verlief reibungslos. Zusätzlich wurde der Stift von einem Schüler als Lesehilfe genutzt, um den Überblick in den Zeilen nicht zu verlieren. Lediglich beim Auftreten technisch bedingter Probleme, wie u.a. Schwierigkeiten beim Auslesen der Audiopunkte vor dunklem Hintergrund und beim Wechseln der Batterien bedurften die Lernenden zusätzlicher Unterstützung.

\section{Selbstständigkeit im Lernprozess}

Alle Sitzungen waren überwiegend durch eine ruhige und arbeitsintensive Atmosphäre geprägt. Mit dem Start der Fördersitzung wählten die Lernenden zügig eigenständig ihre Materialien und begannen mit dem Lesen. Nur vereinzelt kam es zu Unterbrechungen, u.a. wenn sich die Lernenden gegenseitig Geräusche vorspielten, eigene Audioaufnahmen erstellten oder Nachfragen zum Textverständnis stellten. Leider entstand in einer der beiden Kleingruppen bereits zu Beginn durch den Einsatz der Lesepässe eine Wettbewerbsatmosphäre, sodass die Bilderbücher zunächst möglichst schnell und wenig intensiv rezipiert wurden. ${ }^{12}$

Im Verlauf der Sitzungen war zu beobachten, dass alle Lernenden bei der ersten Bilderbuchrezeption den Audiostift vornehmlich zunächst spielerisch einsetzten. Hierzu wurden alle im Buch befindlichen Audiosticker einmal von ihnen angewählt. Teilweise waren auch beim zweiten Lesen des Bilderbuches noch keine vertieften Leseprozesse zu beobachteten. Erst nach dem erneuten Verweis der Lehrperson an Schüler 003 und Schülerin 005, die Audiodateien als Unterstützung zum Lesen der Texte einzusetzen, nahm die ausschliesslich auditive Rezeption des Bilderbuches

11 Lediglich zu Beginn hielten zwei Schülerinnen den Audiostift nicht senkrecht genug, sodass die Informationen auf den Audiostickern nicht ausgelesen werden konnten.

12 Wiederholte Hinweise durch die Lehrperson, die Bilderbücher im eigenen Tempo zu bearbeiten, um nicht nur das Buch mehr zu geniessen, sondern am Ende auch die Aufgaben zum Buch erfolgreich beantworten zu können, entschärfte die Wettbewerbssituation mit Fortschreiten des Projektes. 
allmählich ab. Dennoch kam es im weiteren Verlauf des Projektes immer wieder vor, dass Lernende - insbesondere in Situationen, in denen Textschwierigkeiten auftraten - ihren eigenständigen Leseprozess abbrachen und ausschliesslich mit den Audiodateien arbeiteten. Diese Selbstregulation des Rezeptionsprozesses wird auch von Schülerin $005 \mathrm{im}$ Interview beschrieben:

"Ja paar Mal waren schwierige Wörter ich hab das nicht gekennt und hab das auf Arabisch gehört, danach ich habe das gekennt» (Z: 37-38).

Die Selbstregulation des Rezeptionsprozesses in der offenen Lernumgebung konnte dabei leider nicht bei allen Lernenden beobachtet werden. Beispielhaft sei die Selbststeuerung bei der Arbeit mit der Lernumgebung im Folgenden bei den beiden Schülerinnen 004 (Sprachanfängerin, A1) und Schülerin 006 (fortgeschrittene Sprachanfängerin B1) kontrastiert.

Schülerin 004 streifte in den ersten Sitzungen, trotz der eingangs stets wiederholten mehrsprachigen Einführung zum Lesen der Bilderbücher mit den Audiostiften, mit ihrem Audiostift zunächst durch alle bereitgestellten Bilderbücher. Dabei las sie zunächst die arabischen Textpassagen und wählte anschliessend den dazugehörigen herkunftssprachlichen Audiosticker an. Das Erlesen bzw. die Rezeption der Audiodateien in der Zielsprache wurden bei Schülerin 004 in den ersten drei Sitzungen nicht beobachtet. Wenngleich sie den herkunftssprachlichen Audiodateien sichtlich interessiert folgte, forderten die aufgespielten Fragen und Anregungen sie nicht zu einem interaktiven Rezeptionsprozess heraus. Dass der eingestellte Modus und die in Verbindung damit vorgesehenen Nutzungsweisen und -möglichkeiten der jeweiligen Seite nicht mit ihrer - vor allem auf Erkundung ausgerichteten - Nutzungsweise zusammenpassten, schien sie nur wenig zu stören. Da bis zum Ende der dritten Sitzung kein Moduswechsel dieses oberflächlichen Rezeptionsprozesses in ihrer Herkunftssprache stattfand, war Schülerin 004 mit den digitalisierten Bilderbüchern bereits am Ende der dritten Sitzung fertig. Weiterführende Leseaufgaben wurden nicht von ihr bearbeitet. Nach dem umfassenden Eingreifen der Projektleitung in der vierten Sitzung begann sie erneut mit dem Lesen des ersten Bilderbuches. Obgleich beobachtet werden konnte, dass sie sich für den Leseprozess nun mehr Zeit nahm und Audiodateien wiederholt anspielte, wurden Audiodateien mit Leseaufgaben weiterhin nur vereinzelt von ihr bearbeitet. Immer wieder konnte beobachtet werden, dass Audiosticker nicht gezielt von ihr angewählt worden und das Auffinden von Textstellen zur Beantwortung von Leseaufgaben ausblieb. Es wird vermutet, dass ihre geringen sprachlichen Fertigkeiten in der Zielsprache eine intensivere Auseinandersetzung mit diesen erschwerten und die offene Gestaltung der Lernsituation bzw. die damit einhergehende Wahlfreiheit zur Überforderung führten. 
Während Schülerin 004 nicht selbstständig in eine systematische Nutzung des Audiostiftes wechselte, nutzte Schülerin 006 den Audiostift nach einer ersten explorativen Phase, in der sie sich alle Audiosticker eines Bilderbuches einmal vorspielen liess, zielgerichtet zum Erlesen des Bilderbuches. Dabei begann sie stets mit dem Abspielen der Audiodateien in Deutsch und wechselte dann ins Arabische. Dieses Vorgehen beschreibt sie auch im Interview

«Bei dem Heft Eine Kiste Nichts, ich hab paar Wörter nicht verstanden da hab ich meine Sprache gehört, dann hab ich gut verstanden» (Schülerin 006, Z: 25-26).

Zudem setzte sie den Audiostift ein, um sich anzuhören, wie manche Wörter auf Deutsch ausgesprochen werden. Die Audiobeiträge wurden in diesen Fällen leise von ihr mitgesprochen.

Wird sie zum wiederholten Lesen von Textabschnitten aufgefordert, greift sie nur bei Verständnisschwierigkeiten auf den Audiostift zurück. Obwohl Schülerin 006 einen deutlich sichereren Umgang mit dem Audiostift zeigte, wurden auch von ihr zunächst nicht alle Anregungen zur interaktiven Bilderbuchbetrachtung auf den Audiostickern umgesetzt. Wenngleich explizite Anweisungen wie: «Blättere noch einmal um», «Lies noch einmal nach» oder «Überlege kurz, wie ...» den Audiodateien beigefügt waren, sprang auch Schülerin 006, wie die anderen Lernenden auch, oftmals unmittelbar zum nächsten Audiosticker, ohne sich der Beantwortung der Frage zu widmen. Erst nach Intervention durch die Projektleitung unterbrach Schülerin 006 den Leseprozess, um z.B. alternative Textausgänge zu imaginieren.

Zusammenfassend bestätigt sich damit, wie bereits in einer Vielzahl unterschiedlicher Studien zum offenen Lernen gezeigt wurde, dass die Wirkung offener LehrLern-Formen auf Schülerinnen und Schüler differenziert werden muss. So verweisen Steffens und Höfer (2014) darauf, dass gerade lernschwächere Schülerinnen und Schüler, mit geringem Vorwissen und niedrigem Selbstkonzept Neugelerntes nicht effektiv verarbeiten können, wenn sie zu wenig Ordnungsstruktur und Orientierung in Lernprozessen vorfinden. So führen fehlende Selbststeuerung des Lernprozesses und die unzureichende Lenkung durch die Lehrperson dazu, dass in offenen Unterrichtsphasen Unterrichtszeit auch für nicht unterrichtsrelevante Tätigkeiten genutzt wird. Auch Schüler 003 verweist im Interview auf die Frage nach Gründen zu Schwierigkeiten im Umgang mit den Materialien auf seine fehlende Orientierung: «Weil manchmal ich gucke die Buch, ich lese hier und gucke den Blatt und dann hab ich vergessen wo war ich» (Z: 47-48).

Im Unterricht wurde Schüler 003 folglich auch immer wieder beobachtet, wie er mit der Schnur der Kopfhörer spielte oder vor sich hinstarrte. 


\section{Diskussion der Ergebnisse und Ausblick}

Ausgehend von den Herausforderungen in der Unterrichtsgestaltung in internationalen Sprachklassen wurde in mehreren Design-Zyklen eine Lernumgebung mit mehrsprachigen Bilderbüchern und digitalen Audiostiften entwickelt. Während die Probandinnen und Probanden in den ersten beiden Designzyklen in der Lernumgebung eng pädagogisch begleitet wurden, sollten Lernende im dritten Zyklus eigenständig mit der überarbeiteten Lernumgebung arbeiten. Das Forschungsinteresse galt dabei der Frage, ob die eingesetzten audio-digitalen Medien lesemotivierend wirken und inwieweit sie den mehrsprachigen literalen Rezeptionsprozess unterstützen. Auf Entwicklungsebene sollte kritisch reflektiert werden, ob die Lernenden mithilfe der audio-digitalen Stifte die mehrsprachigen Bilderbücher selbstständig erschliessen können.

Zentrale Ergebnisse der Auswertung seien an dieser Stelle noch einmal zusammengefasst:

- Unabhängig vom Sprachniveau in der Zielsprache Deutsch zeigten alle Lernenden über den Projektzeitraum von 12 Wochen ein grosses Interesse an der Arbeit mit der konzipierten Lernumgebung und eine ausgeprägte Lesemotivation.

- Lernende mit fortgeschrittenen Sprachkompetenzen in der Zielsprache nutzten den Audiostift nach einer ersten explorativen Phase systematisch für die Erschliessung der Bilderbücher, indem sie sich u.a. wiederholt Übersetzungen in ihrer Herkunftssprache zur Sicherung des Textverständnisses und Audiodateien in Deutsch für eine sichere Aussprache der Texte anhörten.

- Lernenden auf A1-Niveau bot das Lernsetting hingegen nicht ausreichend Unterstützungsmöglichkeiten. Auch nach einer ergänzten Buchauswahl, um besonders spracharme Bilderbücher, zeigten diese noch grosse Verständnisschwierigkeiten. Ein selbstständiges Arbeiten mit den Materialien in der Zielsprache konnte trotz der mehrsprachigen Unterstützung der Audiosticker von ihnen noch nicht umgesetzt werden.

- Die auf einen interaktiven Leseprozess ausgelegten Audiosticker wurden erst nach Aufforderung von allen Lernenden intensiver in den Leseprozess eingebunden. Während fortgeschrittene Sprachanfängerinnen und -anfänger die über die Audiosticker eingebrachte Interaktivität des Leseprozesses anschliessend zielgerichtet nutzten, zeigten Lernende mit kurzer Kontaktzeit mit der deutschen Sprache noch grössere Schwierigkeiten.

- Durch die auf Interaktivität ausgerichteten Audiosticker entstand immer wieder das Bedürfnis sich über Textinhalte und Antworten mit den anderen Teilnehmenden auszutauschen, sodass Leseprozesse der Mitschülerinnen und -schüler unterbrochen wurden. 
Entsprechend des iterativen Vorgehens in der Fachdidaktischen Entwicklungsforschung führt der wiederholte Einsatz der Lernumgebung mit empirischer Begleitung zur schrittweisen Anpassung dieser an die vorherrschenden Rahmenbedingungen. Die durch das zyklisch Arbeiten gewonnenen Erkenntnisse auf Theorie- und Praxisebene werden als Designprinzipien (Cobb et al. 2003, 9) (re-)formuliert. Mit der Auswertung der Bedingungen und Wirkungen der eingesetzten Design-Elemente im dritten Zyklus konnten die bereits formulierten handlungsleitenden Maximen des Unterrichtsdesigns: 1. Bilderbuchauswahl unter ganzheitlicher Perspektive, 2. Einbindung der Herkunftssprachen und 3. Entwicklungsproximale Gestaltung authentischer Begleitaufgaben (Dube 2019b, 162f.) bestätigt und erweitert werden.

\section{Design-Prinzip 4: Wechsel individueller und gemeinsamer Leseaktivitäten}

Wie die Beobachtungen im dritten Design-Zyklus belegen, bedarf es bei einer Annährung an schulische Rahmenbedingungen immer wieder Phasen des gemeinsamen Austauschs. So zeigt sich, dass - will man der Prämisse der Übersichtlichkeit nachkommen - einerseits nicht alle Anregungen der sprachlichen und ästhetischen Gestaltung des Bilderbuches über den Audiostift aufgegriffen werden können und andererseits inhaltliche Fragen oder Gesprächsimpulse der Lesenden nicht in eine Gesprächssituation eingebracht werden konnten, sodass es deswegen zu Unterrichtsstörungen kam. Dem hohen Unterhaltungswert mehrsprachiger Bilderbücher, der sich in einer dialogischen Situation wie dem Vorlesen offenbart, konnte die audio-digitale Lernumgebung folglich nicht gerecht werden. Zukünftig sollten deshalb auch immer wieder gemeinsame Lesephasen eröffnet werden, in denen die Lernenden Raum erhalten, Verständnisfragen zu stellen bzw. ihren eigenen Verständnisprozess zu reflektieren und sich über das Gelesene auszutauschen. Hierbei können ebenfalls Aspekte der Sprachintegrativen Förderung aufgegriffen werden. So lädt die Abschlussszene im Bilderbuch «Der Dachs hat heute schlechte Laune» von Moritz Petz (2015), in der die Tiere ausprobieren sollen, wer am «bösesten gucken kann», «wer am unfreundlichsten «Guten Morgen» sagen kann» und «wer am gefährlichsten fauchen kann» (Petz 2015, 23) ${ }^{13}$ nicht nur dazu ein, das Gelesene in der Gruppe umzusetzen, sondern auch die Struktur von Komparativ und Superlativ im Deutschen und anderen Sprachen zu reflektieren. Freie und vorbereitete Gesprächskarten mit Satzanfängen und Wortspeichern könnten die Gespräche in der Gruppe zusätzlich unterstützen.

13 Da die Nummerierung im Bilderbuch fehlt, wurde diese selbst beginnend ab der ersten Textseite vorgenommen. 


\section{Design-Prinzip 5: $\quad$ bewusste Selbststeuerung sprachlicher und literaler Erwerbsprozesse}

Die Ergebnisse des dritten Zyklus haben darüber hinaus verdeutlicht, dass die durch die Bereitstellung mehrsprachiger Materialien angestrebte Individualisierung des Lernprozesses auch zur Überforderung führen kann, wenn die Lernenden nur unzureichend in der Lage sind, ihre sprachlichen und literalen Erwerbsprozesse selbst zu regulieren. So zeigten die Beobachtungen, dass fortgeschrittene Lernende nachhaltig von der Nutzung digitaler Audiostifte zur Rezeption mehrsprachiger Bilderbücher profitierten, während Lernende mit weniger ausgeprägten Sprachkompetenzen in der Zielsprache und wenig Sicherheit in der Steuerung offener Lernprozesse immer wieder auch überfordert waren. Können Lehrpersonen in dialogorientierten Vorlesesituationen spontan auf Verständnisschwierigkeiten und eine fehlende Fokussierung reagieren, sind die Unterstützungsmöglichkeiten des digitalen Audiostiftes in diesem Punkt begrenzt. Bei der Neugestaltung der Audiosticker muss diese Beobachtung eine stärkere Berücksichtigung finden.

Wie die vorangegangenen Einzelsitzungen in den ersten beiden Design-Zyklen bereits vermuten liessen, potenzierte sich der Ressourceneinsatz für die Bereitstellung mehrsprachiger Lernmaterialien mit wachsender Schülerzahl deutlich. Im Projekt konnte der hohe Ressourceneinsatz für die Digitalisierung der mehrsprachigen Bilderbücher durch die enge Arbeit mit mehrsprachigen Studierenden und studentischen Hilfskräften erreicht werden. Alternativ ist zu überlegen, inwieweit mehrsprachige Lehrpersonen, Praktikantinnen und Praktikanten, Eltern oder ältere Schülerinnen und Schüler z.B. im Rahmen einer Projektwoche in die Materialerstellung eingebunden werden könnten.

Wenngleich die ersten empirischen Daten des dritten Design-Zyklus belegen, dass die Nutzung audio-digitaler Stifte zur Rezeption mehrsprachiger Bilderbücher die Lesemotivation in der Zielsprache nachhaltig unterstützen können, sind verallgemeinernde Aussagen kaum möglich. So ist zu vermuten, dass der erfolgreiche Einsatz der Audiostifte nicht nur durch Komplexität der Unterrichtssituation an sich, sondern auch durch Faktoren wie (Lern-)Motivation, Intelligenz und Alter sowie der Sprachlerneignung und Sprachlernstrategien beeinflusst wurden (Heine 2018, 97). Im vierten Zyklus gilt es nun, die aus den vorangegangenen Zyklen erworbenen Erkenntnisse auf Forschungs- und Entwicklungsebene in Form von Design-Prinzipien in die Überarbeitung der Lernumgebung einfliessen zu lassen, um die didaktische Theoriebildung zur Sprachintegrativen Leseförderung mit audio-digital aufbereiteten mehrsprachigen Bilderbüchern beim Einsatz in der Kleingruppe zu finalisieren. 


\section{Literaturverzeichnis}

Ballis, Anja, Claudia Maria Pescher, und Rebecca Schuler, Hrsg. 2019. Mehrsprachige Kinderund Jugendliteratur. Überlegungen zur Systematik, Didaktik und Verbreitung. Baltmannsweiler: Schneider.

Belke, Gerlind. 2003. Mehrsprachigkeit im Deutschunterricht. Sprachspiele. Spracherwerb. Sprachvermittlung. Baltmannsweiler: Schneider.

Belke, Gerlind. 2009. Poesie und Grammatik: Kreativer Umgang mit Texten im Deutschunterricht mehrsprachiger Lerngruppen. Baltmannsweiler: Schneider.

Briddigkeit, Bettina et al. Hrsg. 2004. Deutsch als Zweitsprache - systematisch fördern: Unterrichtsmaterialien für Vorschulkinder und Schulanfänger. Hamburg: Persen.

Cobb, Paul, Jere Confrey, Andrea diSessa, Richard Lehrer, und Leona Schauble. 2003. «Design Experiments in Educational Research». In Educational Researcher, 32(1), 9-13. https://doi. org/10.3102/0013189x032001009.

Dohrn, Antje. 2006. Leseförderung mit literarischen Texten im DaZ-Unterricht: Bausteine für einen integrativen Deutschunterricht. Frankfurt am Main: Peter Lang. https://www.fachportal-paedagogik.de/literatur/vollanzeige.html?FId=901565\#vollanzeige.

Dube, Juliane, und Erkan Gürsoy. 2018. «Mehrsprachige Bilderbücher und audio-digitale Hörstifte. Sprachintegrierte Leseförderung im Unterricht mit neu zugewanderten Schüler/ innen». In Mehrsprachige Leseförderung: Grundlagen und Konzepte, hrsg. v. Sabine Kutzelmann, und Ute Massler, 131-145. Tübingen: Narr. https://www.fachportal-paedagogik.de/ literatur/vollanzeige.html?FId=1148531\#vollanzeige.

Dube, Juliane, Stephan Hussmann. 2019. «Fachdidaktische Entwicklungsforschung (Design Research). Theorie- und empiriegeleitete Gestaltung von Unterrichtspraxis». In Dialogische Verbindungslinien zwischen Theorie und Praxis, hrsg. v. Claudia Priebe, Christiane Mattiesson, Katrin Sommer, 18-37. Bad Heilbrunn: Klinkhardt. https://www.klinkhardt.de/newsite/ media/20190320_9783781522954\%20PriebeMattiesson.pdf.

Dube, Juliane. 2019a. «Fachdidaktische Entwicklungsforschung zwischen Handlungs- und Praxisforschung». In Empirische Erhebungs- und Auswertungsverfahren in der deutschdidaktischen Forschung, hrsg. v. Jan Boelmann. Bd. 2., 49-65. Baltmannsweiler: Schneider. https://www.fachportal-paedagogik.de/literatur/vollanzeige.html?Fld=1097532\#vollanzeige.

Dube, Juliane. 2019b. «Mehrsprachige Bilderbücher als Kontexte zur Sprachintegrativen Leseförderung bei neu zugewanderten Schülerinnen und Schülern». In Sinnstiftende LehrLernprozesse initiieren. Zur Rolle von Kontexten in der Fachdidaktik, hrsg. v. Bernd Ralle, und Jörg Thiele, 147-167. Münster: Waxmann.

Engin, Havva et al. 2004. Kinder lernen Deutsch als zweite Sprache. Prinzipien, Sequenzen, Planungsraster. Minimalgrammatik. Cornelsen: Berlin 2004. https://www.zvab. com/9783589050949/Kinder-lernen-Deutsch-zweite-Sprache-3589050942/plp. 
Gawlitzek, Ira, und Bettina Kümmerling-Meibauer, Hrsg. 2013. Mehrsprachigkeit und Kinderliteratur. Stuttgart: Fillibach bei Klett. https://www.klett-sprachen.de/mehrsprachigkeitund-kinderliteratur/t-1/9783126880183.

Heine, Lena. 2018. «Erkenntnisse aus der Fremd- und Zweitsprachenforschung und didaktische Implikationen mit besonderem Blick auf neu zugewanderte Deutschlernende». In Unterricht mit neu zugewanderten Kindern und Jugendlichen. Interdisziplinäre Impulse für DaF/DaZ in der Schule, hrsg. v. Verena Harboe, Mirka Mainzer-Murrenhoff, Lena Heine, 81104. Münster, New York: Waxmann.

Hoffmann, Reinhild, und Ingrid Weis. 2011. Deutsch als Zweitsprache - alle Kinder lernen Deutsch. Sprachenlernen in mehrsprachigen Lerngruppen. Praxisorientierte Ansätze der Sprachförderung. Für alle Jahrgangsstufen. Cornelsen: Berlin. https://www.cornelsen. de/produkte/lehrerbuecherei-grundschule-deutsch-als-zweitsprache-alle-kinder-lernendeutsch-2-auflage-sprachenlernen-in-mehrsprachigen-lerngruppen-praxisorientierte-ansaetze-der-sprachfoerderung-fuer-alle-jahrgangsstufen-buch-9783589051557.

Hüttis-Graff, Petra. 2008. «Vom Hören zum Lesen - Literarisches Lernen mit Lese- Hör-Kisten». In Medien als Erzählanlass. Wie lernen Kinder im Umgang mit alten und neuen Medien? hrsg. v. Petra Wieler, Petra, 2. Aufl., 105-123. Freiburg: Fillibach.

Kalkavan-Aydin, Zeynep, Hrsg. 2015. Deutsch als Zweitsprache. Didaktikfür die Grundschule. Cornelsen: Berlin. https://www.cornelsen.de/produkte/fachdidaktik-fuer-die-grundschuledeutsch-als-zweitsprache-2-auflage-didaktik-fuer-die-grundschule-buch- 9783589163960.

Kruse, Iris. 2012. Kinder- und Jugendliteratur intermedial erfahren, erleben, lesen. Baltmannsweiler: Schneider.

Meuser, Michael, und Ulrike Nagel. 2009. «Das Experteninterview - konzeptionelle Grundlagen und methodische Anlage». In Methoden der vergleichenden Politik- und Sozialwissenschaft. hrsg. v. Susanne Pickel et al. Wiesbaden: VS-Verlag.

Möbius, Thomas. 2008. «Das literarische Sehgespräch als sprachlich-kommunikative Vermittlungsweise bilddominierter Medienangebote». In LOGIN! Kreativer Deutschunterricht und neue Medien. hrsg. v. Volker Frederking, Matthis Kepser, Matthias Rath, 141-156. München: kopaed.

Möller, Jens, und Ulrich Schiefele. 2004. «Motivationale Grundlagen der Lesekompetenz». In Struktur, Entwicklung und Förderung von Lesekompetenz. Vertiefende Analysen im Rahmen von PISA 2000. hrsg. v. Ulrich Schiefele et al., 101-124. Wiesbaden: VS Verlag für Sozialwissenschaften.

Petko, Dominik, Monika Waldis, Pauli Christine, und Kurt Reusser. 2003. «Methodologische Überlegungen zur videogestützten Forschung in der Mathematikdidaktik» Zeitschrift für die Didaktik der Mathematik, 35 (6), 65-280. https://www.ife.uzh.ch/dam/jcr:000000003212-6146-ffff-ffff9416ba0d/ZDM_06_2003_petko_waldis.pdf. 
Ockel, Eberhard. 2000. Vorlesen als Aufgabe und Gegenstand des Deutschunterrichts. Baltmannsweiler: Schneider. https://www.fachportal-paedagogik.de/literatur/vollanzeige. html?Fld=801655\#vollanzeige.

Spinner, Karl H. 2004. «Gesprächseinlagen beim Vorlesen». In Kein endgültiges Wort. Die Wiederentdeckung des Gesprächs im Literaturunterricht. hrsg. v. Gerhard Härle, und Marcus Steinbrenner, 291-307. Baltmannsweiler: Schneider. https://www.fachportal-paedagogik. de/literatur/vollanzeige.html?Fld=681706\#vollanzeige.

Steffens, Ulrich, und Dieter Höfer. 2014. Die Hattie-Studie. http://www.sqa.at/pluginfile. php/813/course/section/373/hattie_studie.pdf.

Wieler, Petra. 1997: Vorlesen in der Familie: Fallstudien zur literarisch-kulturellen Sozialisation von Vierjährigen. Weinheim: Juventa. https://www.fachportal-paedagogik.de/literatur/ vollanzeige. $h t m l$ ?FId=466672\#vollanzeige. 